\title{
Revealing the Dynamics and Roles of Iron Incorporation in Nickel Hydroxide Water Oxidation Catalysts
}

Chunguang Kuai ${ }^{1,2,3}$, Cong $\mathrm{Xi}^{2, \#}$, Anyang $\mathrm{Hu}^{3, \#}$, Yan Zhang ${ }^{2,4}$, Zhengrui $\mathrm{Xu}^{3}$, Dennis Nordlund ${ }^{4}$, Cheng-Jun Sun ${ }^{5}$, Christopher A. Cadigan 6 , Ryan M. Richards ${ }^{6}$, Luxi Li $^{5 *}$, Cun-Ku Dong ${ }^{2}, \mathrm{Xi}-\mathrm{Wen} \mathrm{Du}^{2 *}$, Feng Lin ${ }^{3 *}$

\section{Affiliations}

1 School of Electrical Engineering and Automation, Wuhan University, Wuhan 430072, China.

2 Institute of New-Energy Materials, School of Materials Science and Engineering, Tianjin University, Tianjin, 300072, China. Email: xwdu@tju.edu.cn

3 Department of Chemistry, Virginia Tech, Blacksburg, VA 24061, USA. Email: fenglin@vt.edu

4 Stanford Synchrotron Radiation Lightsource, SLAC National Accelerator Laboratory, Menlo Park, California 94025, USA.

5 Advanced Photon Source, Argonne National Laboratory, Argonne, Illinois 60439, USA. Email: luxili@anl.gov

6 Department of Chemistry, Colorado School of Mines, Golden, Colorado 80401, USA.

\# These authors contribute equally to this work. 


\section{Experimental section}

Synthesis of $\mathrm{Ni}(\mathrm{OH})_{2}$. The $\mathrm{Ni}(\mathrm{OH})_{2}$ nanosheets were synthesized according to the method reported elsewhere ${ }^{1,2}$. The details are as follows. $\mathrm{Ni}\left(\mathrm{NO}_{3}\right)_{2} \bullet 6 \mathrm{H}_{2} \mathrm{O}$, urea and benzyl alcohol were mixed with the weight ratio of 2:1:4 and then dissolved in $50 \mathrm{~mL}$ methanol with vigorous stirring for 1 hour. The solution was then transferred to an autoclave. Before heating, the mixture was purged with Ar for 1 min. Ar was filled to a pressure of 9 bars. Then the mixture was heated to $265^{\circ} \mathrm{C}$ and maintained for $1.5 \mathrm{~h}$. The vapor was then vented, and a green powder was collected. The powder was washed thoroughly with isopropanol and DI water and finally dried in a vacuum oven at $100^{\circ} \mathrm{C}$ overnight.

Electrochemical measurement. The electrode was prepared through a dip casting method. First, $0.1 \mathrm{mg}$ catalyst was dispersed in $0.5 \mathrm{ml}$ water, and then mixed with 0.5 $\mathrm{ml}$ methanol and $20 \mu \mathrm{l}$ Nafion. The mixtures were ultrasonically dispersed for about 3 hours to form a catalyst ink. $20 \mu \mathrm{l}$ of catalyst ink was carefully dropped onto a $0.5^{*} 0.4$ $\mathrm{cm}^{2}$ carbon paper electrode, with a mass loading of about $0.01 \mathrm{mg} / \mathrm{cm}^{2}$. Then the catalyst-coated electrodes were dried at room temperature for about $1 \mathrm{~h}$. We then conducted CV measurements using a Biologic SP150 potentiostat in an $\mathrm{O}_{2}$ saturated $1 \mathrm{M} \mathrm{KOH}$ electrolyte. A graphite rod and a $\mathrm{Ag} / \mathrm{AgCl}$ electrode are used as the counter and reference electrodes, respectively. The following equation is used to convert the applied potential between $\mathrm{Ag} / \mathrm{AgCl}$ and reversible hydrogen electrode (RHE):

$$
\mathrm{E}(\mathrm{RHE})=\mathrm{E}(\mathrm{Ag} / \mathrm{AgCl})+0.059 \mathrm{pH}+0.197 \mathrm{~V}
$$

The scan rate for the $\mathrm{CV}$ test is set to be $10 \mathrm{mV} \mathrm{s}^{-1}$.

TEM and SEM Characterization. The morphologies of the materials and electrode 
were investigated using a transmission electron microscopy (TEM) (JEOL JEM 2100 (HRP): Thermal emitter (LaB6) scanning TEM) with an accelerating voltage of $200 \mathrm{kV}$ and a field-emission scanning electron microscopy (SEM) (LEO 1550) with an accelerating voltage of $5 \mathrm{kV}$ at the Nanoscale Characterization and Fabrication Laboratory of Virginia Tech.

XAS measurements. All the soft XAS measurements were carried out at the Stanford Synchrotron Radiation Lightsource, SLAC National Accelerator Laboratory. Soft XAS for Ni L-edge and O K-edge was performed at Beam Line 10-1. Samples for ex-situ soft XAS were mounted on a $3 / 4$ inch Al stick at an angle of about $55^{\circ}$ with respect to the incident beam. A $1,0001 \mathrm{~mm}^{-1}$ spherical grating monochromator with $20 \mu \mathrm{m}$ entrance and exit slits were applied to provide $\sim 10^{11} \mathrm{ph} \mathrm{s}^{-1}$ at $0.2 \mathrm{eV}$ resolution in a 1 $\mathrm{mm}^{2}$ beam spot.

All the hard XAS were measured at beamline 20-ID at the Advanced Photon Source, Argonne National Laboratory. For all ex-situ experiments, samples were prepared on carbon paper (Toray Carbon Paper 090, The Fuel Cell Store) in a manner similar to that for electrochemical tests, removing Nafion to prevent undesired interference. The $\mathrm{Ni}$ K-edge spectra were collected in the fluorescence mode with the samples aligned at an angle of $\sim 45^{\circ}$ with respect to the incident beam and the Lytle detector. To minimize the background from elastic and Compton scattering, Z-1 filters (e.g. Co filter for Ni Kedge) with Soller slits were placed before the window of the detector. Pure metal foils were used to align $\mathrm{E}_{0}$ to $8333 \mathrm{eV}$ for $\mathrm{Ni}$.

Synchrotron X-ray fluorescence microscopy (XFM) The samples were prepared by 
the same method as described in the electrochemical measurement. XFM was measured at the 2-ID-E beamlines at the Advanced Photon Source, Argonne National Laboratory. The samples are raster-scanned by a sub-micrometer $(\sim 700 \mathrm{~nm})$ focused $10 \mathrm{keV}$ X-ray beam with a step size of $0.5 \mu \mathrm{m}$ (fine scan) and $30 \mu \mathrm{m}$ (coarse scan). The fluorescent X-rays are detected by a four-element Si-drift Vortex detector. The schematic of the experimental setup can be found in reference ${ }^{3}$. The raw data is then processed and quantified with MAPS

DFT calculations The DFT calculations were carried out by the Vienna Ab initio Simulation Package (VASP). ${ }^{5,6}$ The nuclei-electron and the electron exchangecorrelation interactions were described by the projector augmented wave (PAW) potentials ${ }^{7}$ and the generalized gradient approximation $(\mathrm{GGA})^{8}$ with the Perdew-BurkeErnzerhof (PBE) ${ }^{9}$ functional, respectively. The van der Waals(vdW) effects were applied through the Grimme's DFT-D3 correction method. ${ }^{10}$ All calculations were spin polarized. The wave functions of valence electrons were expanded by plane wave basis set with a cut off energy of $500 \mathrm{eV}$. The convergence criteria for energy and force during geometrical optimization were set to $10-5 \mathrm{eV}$ and $0.02 \mathrm{eV} / \mathrm{A}^{\circ}$, respectively. The vacuum space of $15 \AA \AA$ was applied to avoid the interactions along the z-direction while the layer space was set $7 \AA$ according to experiment experience18. The Brillouin zone sampling was conducted using a Monkhorst Pack grid of $3 \times 3 \times 1$ k-points mesh grid. ${ }^{11}$ Throughout the calculation, an implicit solvent model was also used to simulate the water solvent environment as implemented in VASPsol. ${ }^{12}$ 

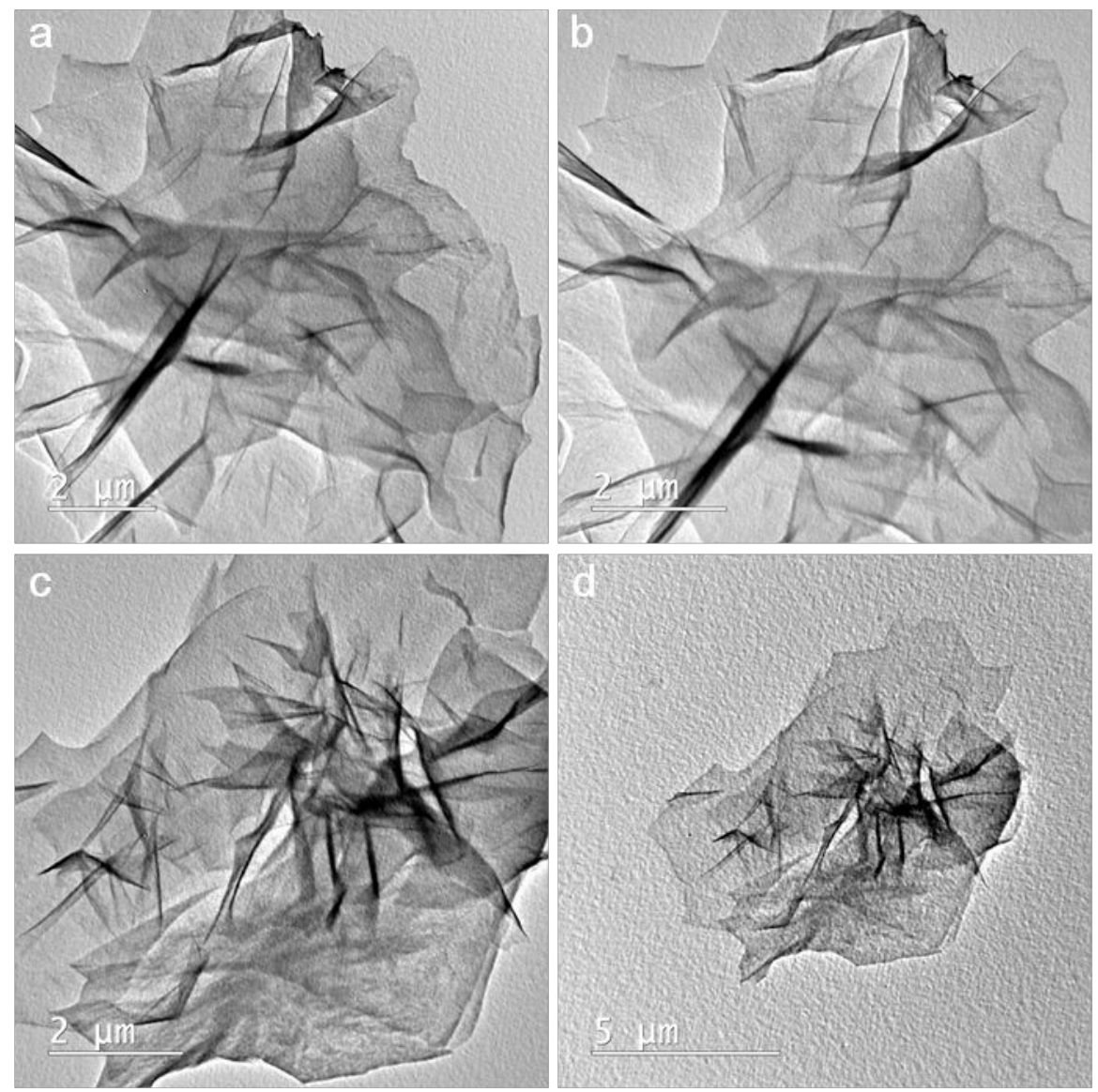

Figure S1. TEM images of the as-prepared $\mathrm{Ni}(\mathrm{OH})_{2}$ nanosheets. The diameter of the nanosheets is around 7-12 $\mu \mathrm{m}$. 

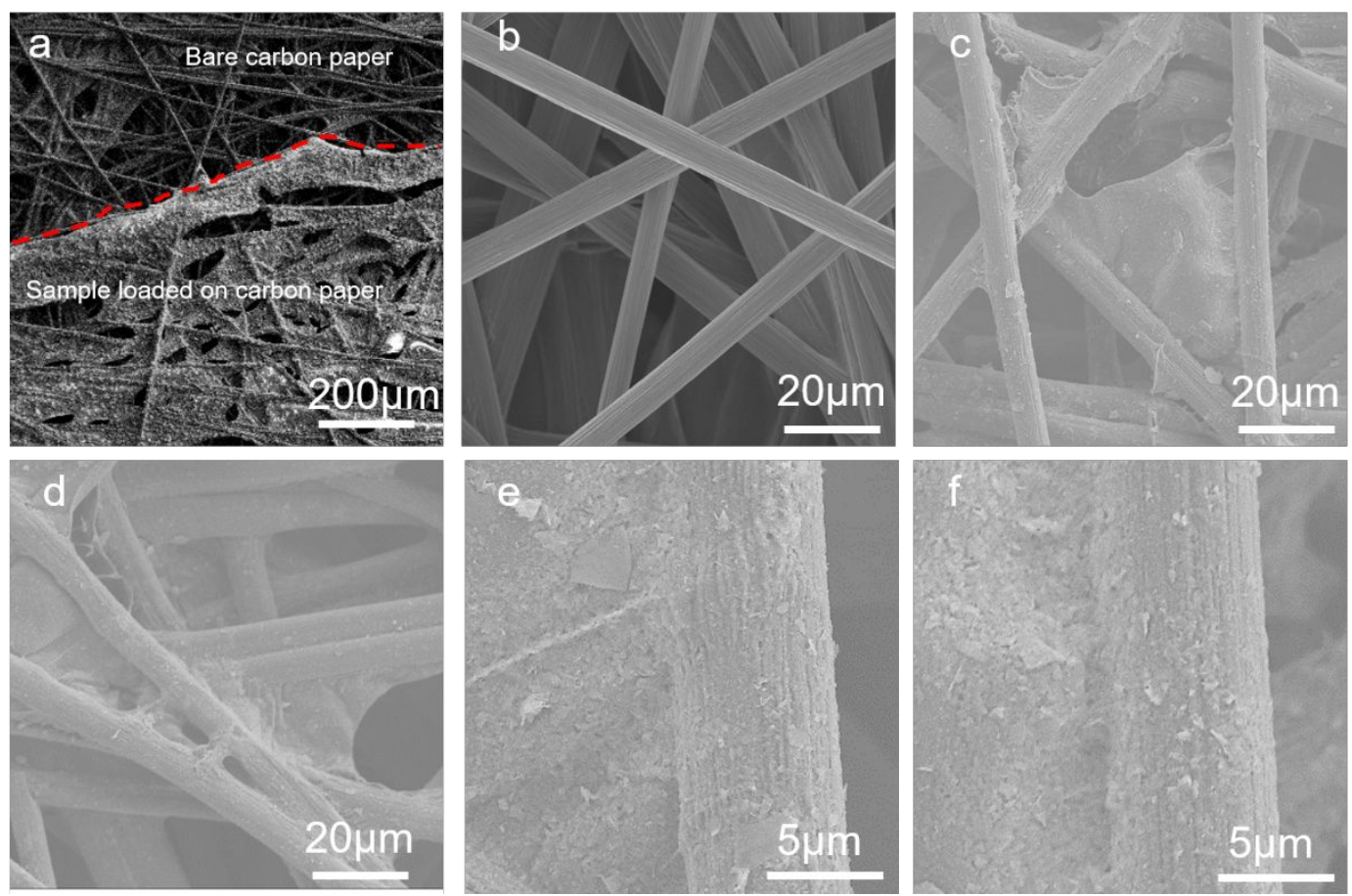

Figure S2. Morphology of the bare and sample loaded carbon paper by scanning electron microscopy (SEM). a) Low magnification images of the sample loaded carbon paper. b) Morphology of the bare carbon paper. c, d, e, f) Morphology of the sample loaded carbon paper. 


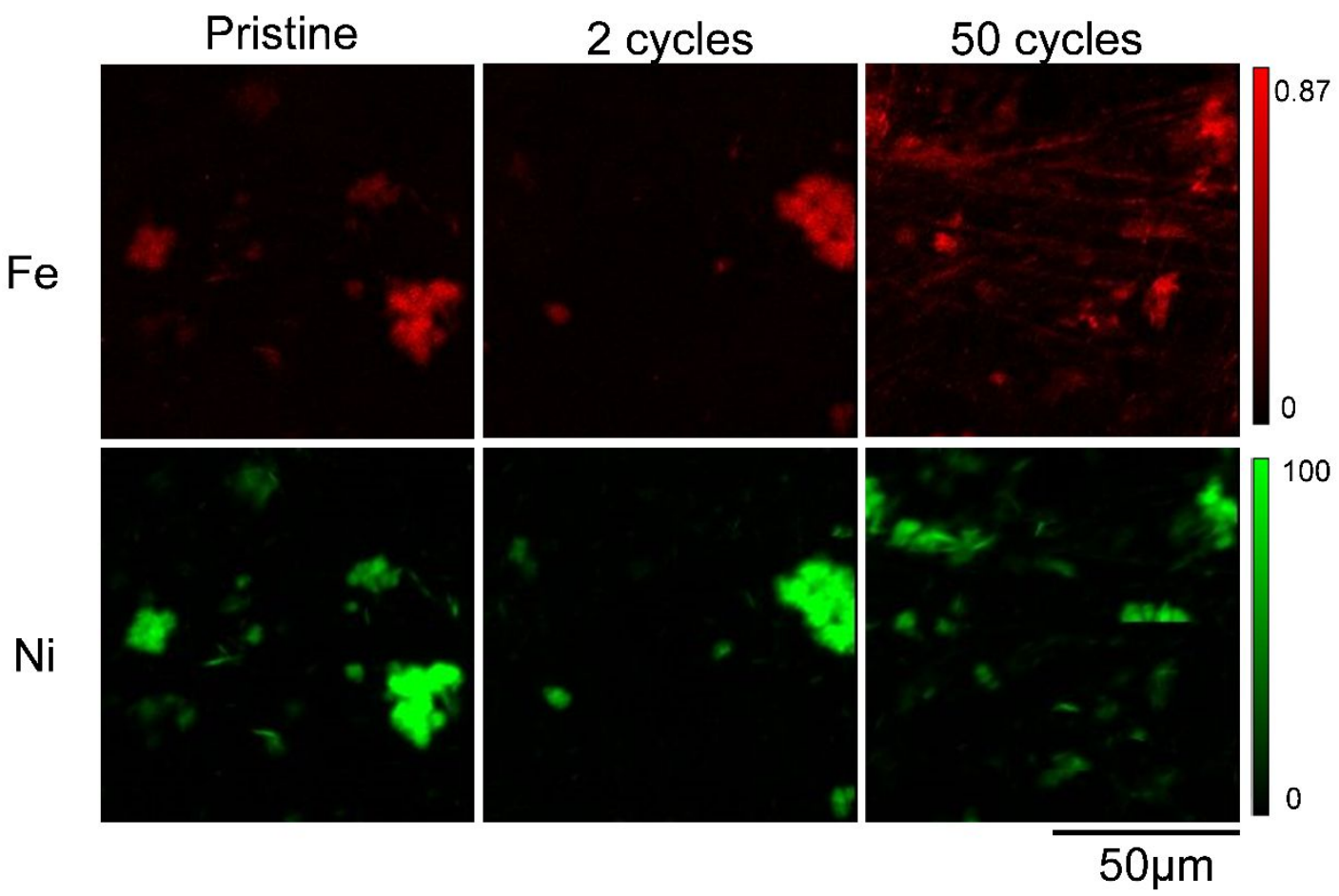

Figure S3. Element mapping for the pristine and cyclic voltammetry (CV) cycled samples by high resolution synchrotron X-ray fluorescence microscopy. The CV cycles are conducted in the range of 1.03-1.73 V vs. RHE in a $1 \mathrm{M} \mathrm{KOH}$ electrolyte containing $10 \mu \mathrm{M} \mathrm{Fe}^{3+}$, with a scan rate of $10 \mathrm{mV} / \mathrm{s}$. The units of the color bar are $\mu \mathrm{g} / \mathrm{cm}^{2}$. The results here show the presence of $\mathrm{Fe}$ in the $\mathrm{Ni}$ hydroxide matrix and the relative concentration maps shown in the manuscript demonstrate the preferred distribution of Fe. 


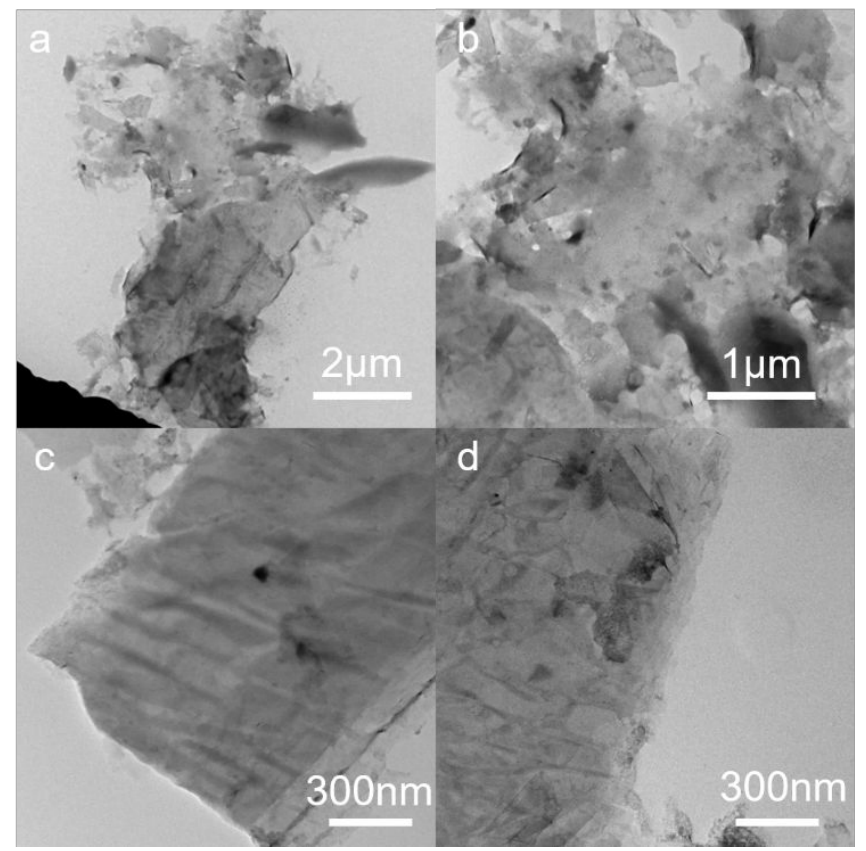

Figure S4. TEM images of the $\mathrm{Ni}(\mathrm{OH})_{2}$ nanosheets after 6 hours CA measurements under $1.6 \mathrm{~V}$ vs. RHE in the Fe containing electrolyte. There is minor presence of Nafion in the sample collected from the used electrode. 


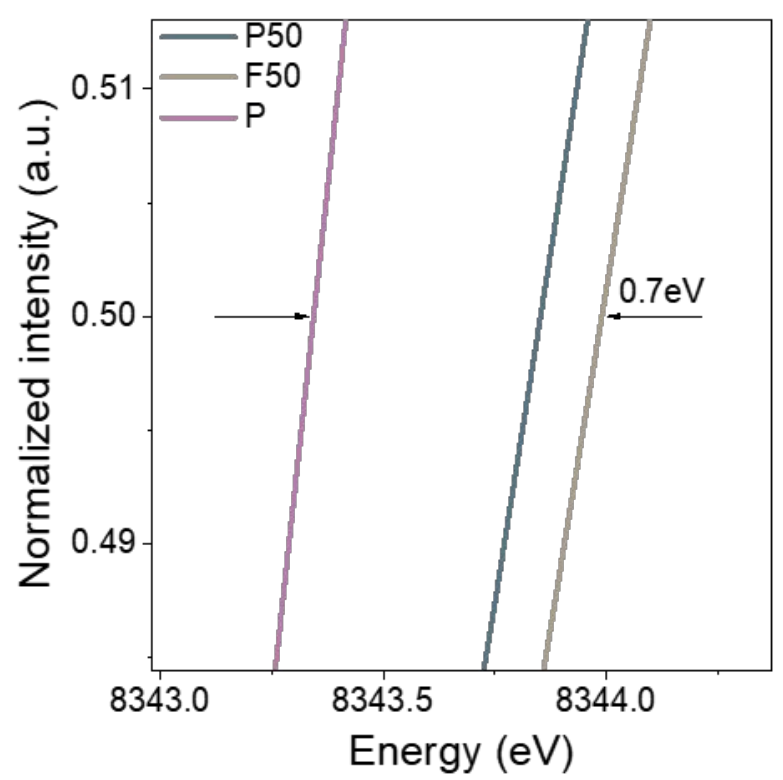

Figure S5. Enlargement of the Ni K-edge of the pristine and iron incorporated $\mathrm{Ni}(\mathrm{OH})_{2}$ nanosheets. $\mathrm{P}$ is pristine material, $\mathrm{P} 50$ is the pristine material after $50 \mathrm{CV}$ cycles, and then a CA measurement under $1.6 \mathrm{~V}$ for $10 \mathrm{~min}$ in purified $1 \mathrm{M} \mathrm{KOH}, \mathrm{F} 50$ is the pristine material after $50 \mathrm{CV}$ cycles in $1 \mathrm{M} \mathrm{KOH}$ containing $10 \mu \mathrm{M} \mathrm{Fe}^{3+}$ and then a CA measurement under $1.6 \mathrm{~V}$ for $10 \mathrm{~min}$ in purified $1 \mathrm{M} \mathrm{KOH}$. 


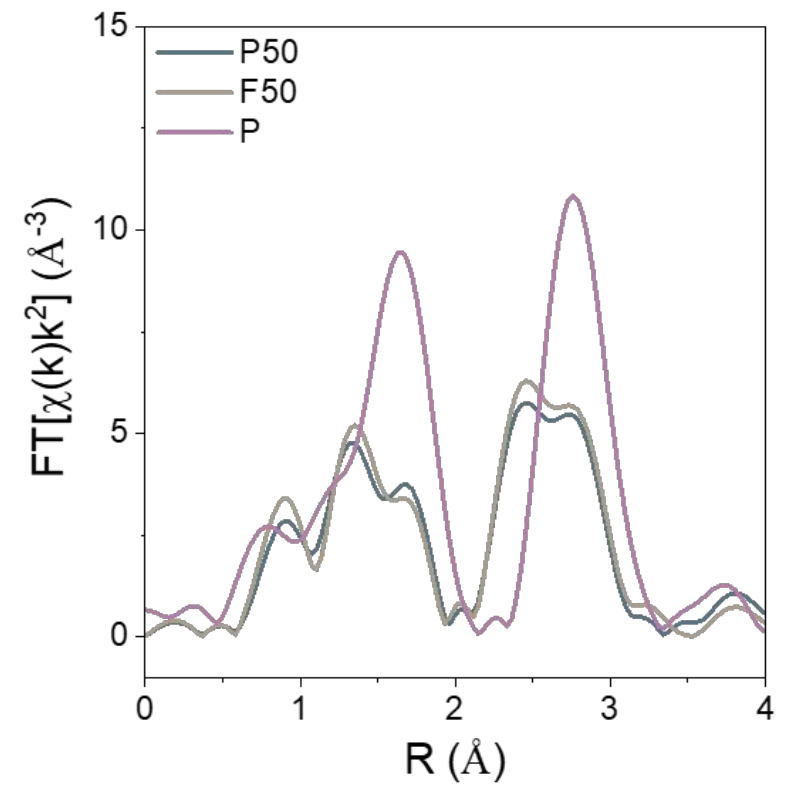

Figure S6. $\mathrm{k} 2$ weighted EXAFS in R space. $\mathrm{P}$ is pristine material, $\mathrm{P} 50$ is the pristine material after $50 \mathrm{CV}$ cycles, and then a $\mathrm{CA}$ measurement at $1.6 \mathrm{~V}$ for $10 \mathrm{~min}$ in purified $1 \mathrm{M} \mathrm{KOH}, \mathrm{F} 50$ is the pristine material after $50 \mathrm{CV}$ cycles in $1 \mathrm{M} \mathrm{KOH}$ containing $10 \mu \mathrm{M}$ $\mathrm{Fe}^{3+}$ and then a CA measurement at $1.6 \mathrm{~V}$ for $10 \mathrm{~min}$ in purified $1 \mathrm{M} \mathrm{KOH}$. 
a

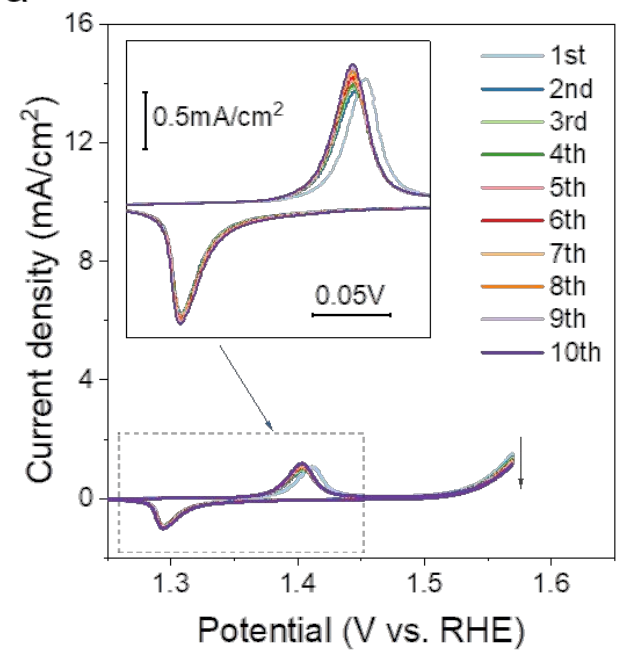

b

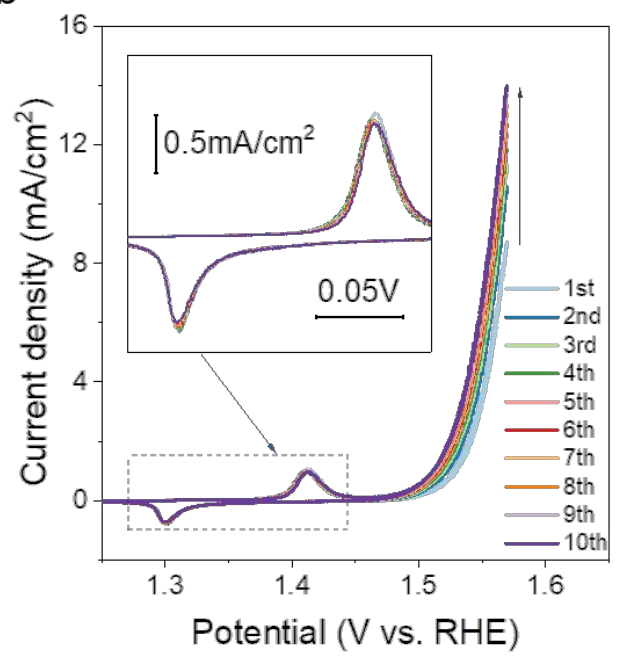

Figure S7. The first $10 \mathrm{CV}$ cycles of the $\mathrm{Ni}(\mathrm{OH})_{2}$ in a) $1 \mathrm{M} \mathrm{KOH}$, b) $1 \mathrm{M} \mathrm{KOH}$ with $10 \mu \mathrm{M} \mathrm{Fe}^{3+}$. The $\mathrm{CV}$ scan rate is $10 \mathrm{mV} / \mathrm{s}$. 

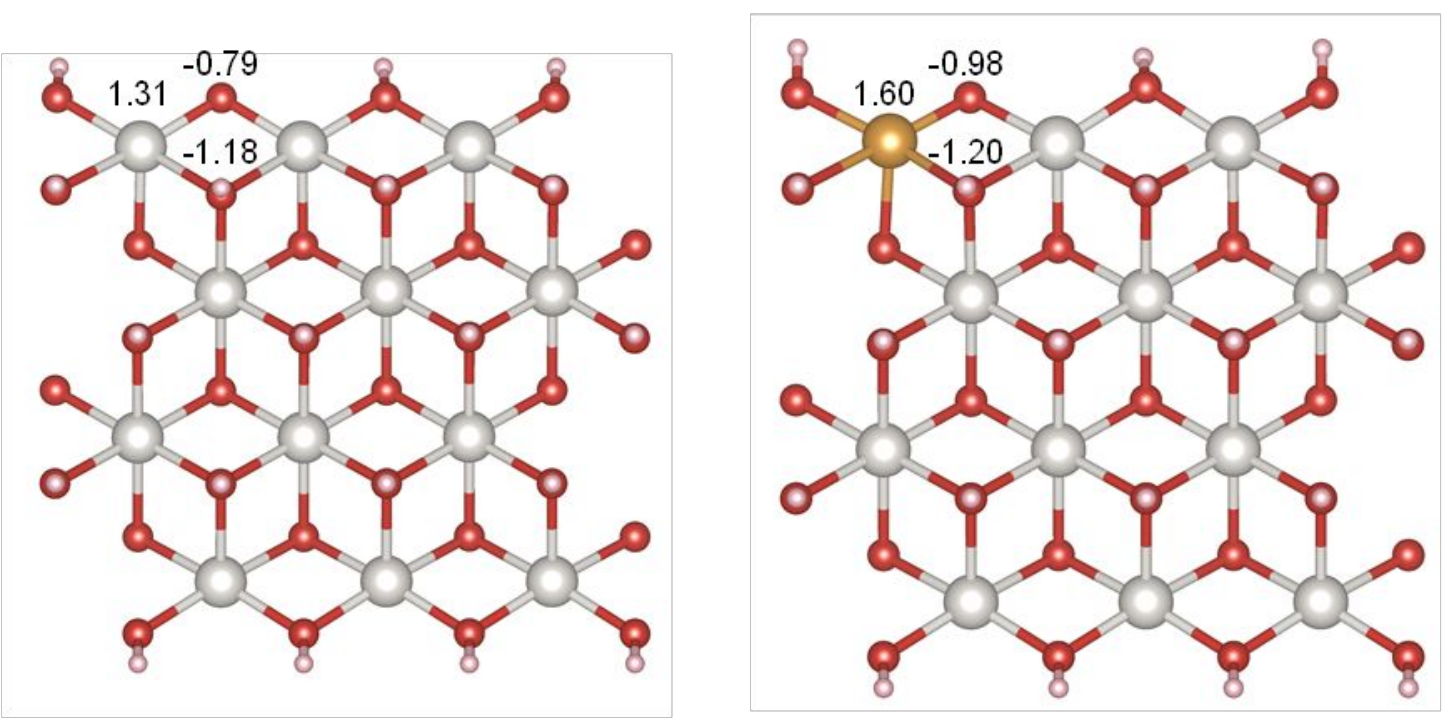

Figure S8. Bader charge calculations of the $\mathrm{Ni}(\mathrm{OH})_{2}$ with and without $\mathrm{Fe}$ modification. The number in the figure are denoted the valance state calculated from Bader charge. Upon $\mathrm{Fe}$ incorporation, the valence state of the $\mathrm{O}$ that bridges $\mathrm{Ni}$ and $\mathrm{Fe}$ shows a pronounced decrease. The gray, gold, red, pink color balls represent $\mathrm{Ni}, \mathrm{Fe}, \mathrm{O}$, and $\mathrm{H}$.

\section{Reference}

(1) Lin, F.; Nordlund, D.; Weng, T.-C.; Zhu, Y.; Ban, C.; Richards, R. M.; Xin, H. L. Phase Evolution for Conversion Reaction Electrodes in Lithium-Ion Batteries. Nat. Commun. 2014, 5 (1), 3358.

(2) He, K.; Lin, F.; Zhu, Y.; Yu, X.; Li, J.; Lin, R.; Nordlund, D.; Weng, T.-C.; Richards, R. M.; Yang, X.-Q.; Doeff, M. M.; Stach, E. A.; Mo, Y.; Xin, H. L.; $\mathrm{Su}, \mathrm{D}$. Sodiation Kinetics of Metal Oxide Conversion Electrodes: A Comparative Study with Lithiation. Nano Lett. 2015, 15 (9), 5755-5763.

(3) de Jonge, M. D.; Vogt, S. Hard X-Ray Fluorescence Tomography - an Emerging Tool for Structural Visualization. Curr. Opin. Struct. Biol. 2010, 20 (5), 606-614.

(4) Vogt, S. MAPS : A Set of Software Tools for Analysis and Visualization of 3D X-Ray Fluorescence Data Sets. J. Phys. IV Proc. 2003, 104, 635-638.

(5) Kresse, G.; Furthmüller, J. Efficiency of Ab-Initio Total Energy Calculations for Metals and Semiconductors Using a Plane-Wave Basis Set. Comput. Mater. Sci. 1996, 6 (1), 15-50.

(6) Kresse, G.; Furthmüller, J. Efficient Iterative Schemes for Ab Initio Total-Energy Calculations Using a Plane-Wave Basis Set. Phys. Rev. B 1996, 54 (16), 11169 11186.

(7) Blöchl, P. E. Projector Augmented-Wave Method. Phys. Rev. B 1994, 50 (24), 17953-17979.

(8) Perdew, J. P.; Burke, K.; Ernzerhof, M. Generalized Gradient Approximation Made Simple. Phys. Rev. Lett. 1996, 77 (18), 3865-3868.

(9) Perdew, J. P.; Ernzerhof, M.; Burke, K. Rationale for Mixing Exact Exchange 
with Density Functional Approximations. J. Chem. Phys. 1996, 105 (22), 99829985.

(10) Grimme, S.; Antony, J.; Ehrlich, S.; Krieg, H. A Consistent and Accurate $A b$ Initio Parametrization of Density Functional Dispersion Correction (DFT-D) for the 94 Elements H-Pu. J. Chem. Phys. 2010, 132 (15), 154104.

(11) Monkhorst, H. J.; Pack, J. D. Special Points for Brillouin-Zone Integrations. Phys. Rev. B 1976, 13 (12), 5188-5192.

(12) Mathew, K.; Sundararaman, R.; Letchworth-Weaver, K.; Arias, T. A.; Hennig, R. G. Implicit Solvation Model for Density-Functional Study of Nanocrystal Surfaces and Reaction Pathways. J. Chem. Phys. 2014, 140 (8), 084106. 